\title{
Mixed large cell neuroendocrine carcinoma and mucosa-associated lymphoid tissue lymphoma of the lung: A case report
}

\author{
TAKESHI MATSUMOTO ${ }^{1}$, YUKIHIRO IMAI ${ }^{2}$, YASUHIRO KOSAKA ${ }^{3}$, \\ TAKASHI SHINTANI $^{3}$ and KEISUKE TOMII ${ }^{1}$ \\ Departments of ${ }^{1}$ Respiratory Medicine, ${ }^{2}$ Pathology and ${ }^{3}$ Radiation Therapy, \\ Kobe City Medical Center General Hospital, Kobe, Hyogo 650-0047, Japan
}

Received April 19, 2014; Accepted February 10, 2015

DOI: $10.3892 / \mathrm{ol} .2015 .2994$

\begin{abstract}
In the present study, a 79-year-old male was referred to Kobe City Medical Center General Hospital due to an abnormal shadow that was revealed on a chest X-ray. The patient possessed a five-year history of consolidation in the right lower lobe, which was diagnosed as chronic aspiration pneumonia and followed up. However, the abnormal shadow adjacent to the pleura gradually increased in size and a novel mass appeared in the right lower lobe that rapidly increased in size. A repeat biopsy revealed a combination of large cell neuroendocrine carcinoma with a clinical tumor-node-metastasis (cTNM) stage of cT2bN2M0 (stage 3A) and mucosa-associated lymphoid tissue lymphoma at Ann Arbor stage 1E. Chemoradiotherapy markedly affected the lesion and the size of the mass was significantly reduced subsequent to four cycles of chemotherapy, which was considered to be a near complete response. The present study reports an extremely rare combination of tumors. The disease course was followed over a period of six years, which included the onset of disease, and the present case may therefore be valuable in clarifying the mechanism of lung cancer development.
\end{abstract}

\section{Introduction}

Mucosa-associated lymphoid tissue (MALT) lymphoma is a low-grade B cell lymphoma that was first identified by Isaacson and Wright (1). Primary pulmonary lymphoma is rare and accounts for $0.5 \%$ of lung tumors, with $72-90 \%$ of pulmonary lymphomas being MALT lymphomas $(2,3)$. The outcome of pulmonary MALT lymphoma is generally favorable, with a five-year survival rate of $>80 \%$ and a median survival time of $>10$ years (4). At present, no consensus exists with regard to treatment, however, simple clinical monitoring

Correspondence to: Dr Takeshi Matsumoto, Department of Respiratory Medicine, Kobe City Medical Center General Hospital, 2-1-1 Minatojima Minamimachi, Kobe, Hyogo 650-0047, Japan

E-mail: fu_rinkazan0202@yahoo.co.jp

Key words: large cell neuroendocrine carcinoma, mucosa-associated lymphoid tissue lymphoma, mix, chemoradiotherapy is recommended $(5,6)$. Surgical resection is usually performed in patients with localized lesions, whereas chemotherapy is administered to patients with bilateral or extrapulmonary involvement, relapse or progression (4). Large cell neuroendocrine carcinoma (LCNEC) is a tumor that was first proposed by Travis et al (7). LCNEC is also rare, accounting for $2.4 \%$ of lung cancers, and its prognosis is extremely poor with a five-year survival rate of $15-57 \%$, and $27-67 \%$ in patients with stage I disease according to TNM staging $(8,9)$. Surgical resection alone is not sufficient for the treatment of LNEC and thus, adjuvant chemotherapy is recommended after surgery even in patients with stage IA disease accordign to TNM staging (10). The present study reports a case of a patient with combined LCNEC and MALT lymphoma that responded well to chemoradiotherapy. The combination of these two tumors is extremely rare, and their development was documented over a period of six years, including the onset of disease. The present study may therefore be valuable in clarifying the mechanism of the development of lung cancer.

\section{Case report}

A 79-year-old male was referred to Kobe City Medical Center General Hospital (Kobe, Japan) with an abnormal shadow that was revealed on a chest X-ray. The patient possessed a history of cerebral infarction, which occurred at 55 years old, had undergone a subtotal gastrectomy for gastric cancer at the age of 70, and had also undergone an aortic arch replacement for thoracic aortic aneurysm at 75 years old. The patient was an ex-smoker, and had not experienced apparent asbestos or silica dust exposure. Follow-up had been performed at Rokko Island Hospital (Kobe, Japan) for the five years prior to the referral (Fig. 1A). Medical attention had been sought at Rokko Island Hospital three years prior to referral for hemoptysis, and a consolidation in the right lower lung field was identified at that time (Fig. 1B). The consolidation was followed up as chronic aspiration pneumonia. Bronchoscopy was performed two years prior to the current admission as the consolidation adjacent to the pleura had enlarged (Fig. 1C), but no specific findings were noted. The consolidation was considered to be chronic aspiration pneumonia since the size varied over time, but it had gradually increased in size in the five years prior to the referral. The patient was referred to Kobe City Medical 

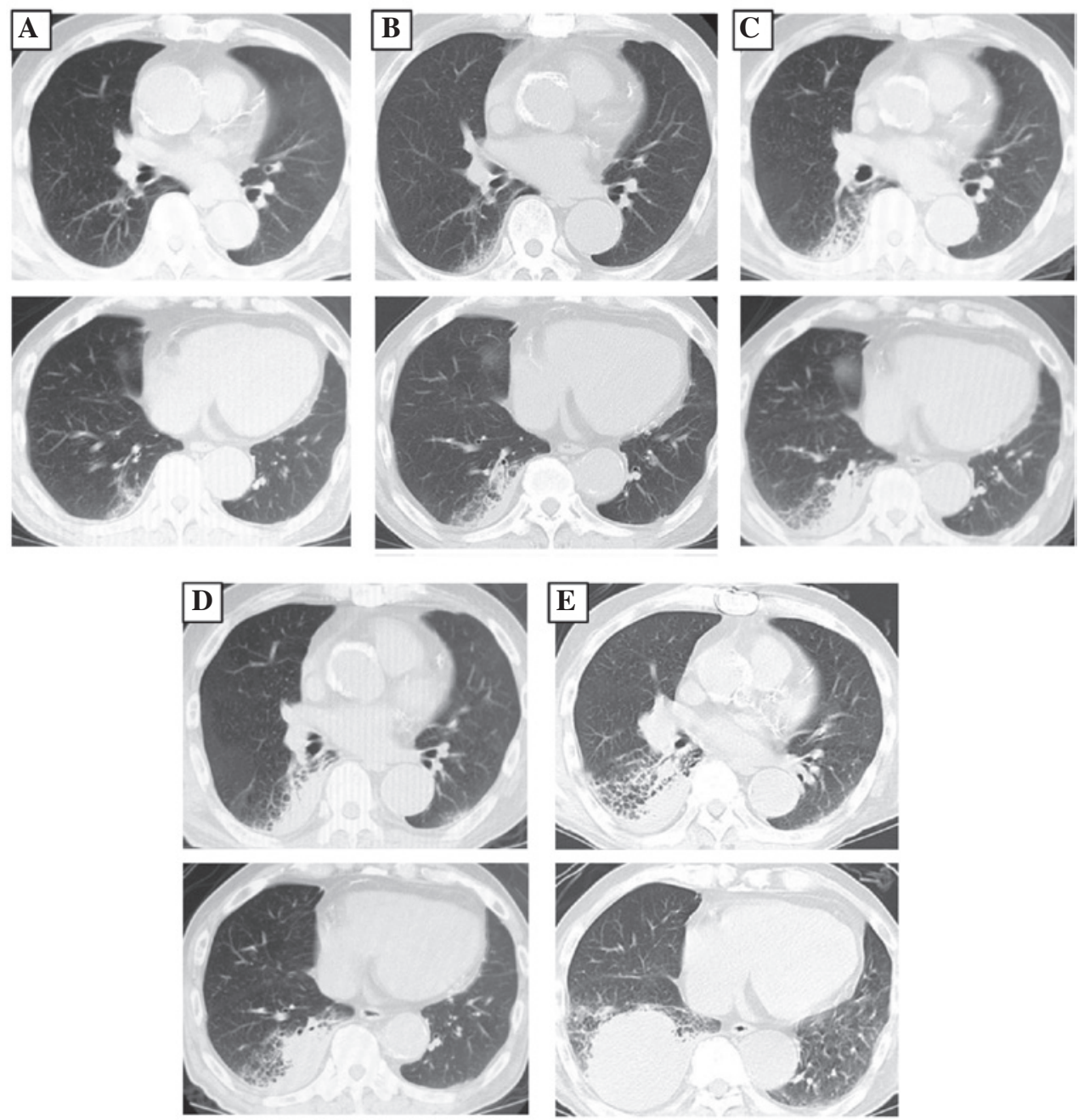

Figure 1. Radiological appearance of the advancing abnormalities in the computed tomography scan of the chest. The images were obtained (A) five years prior to the referral, (B) three years prior to the referral, (C) two years prior to the referral, (D) six months prior to the referral, and (E) at the referral. Consolidation in the right lower lobe gradually became advanced, and the mass grew up quickly immediately prior to the referral.
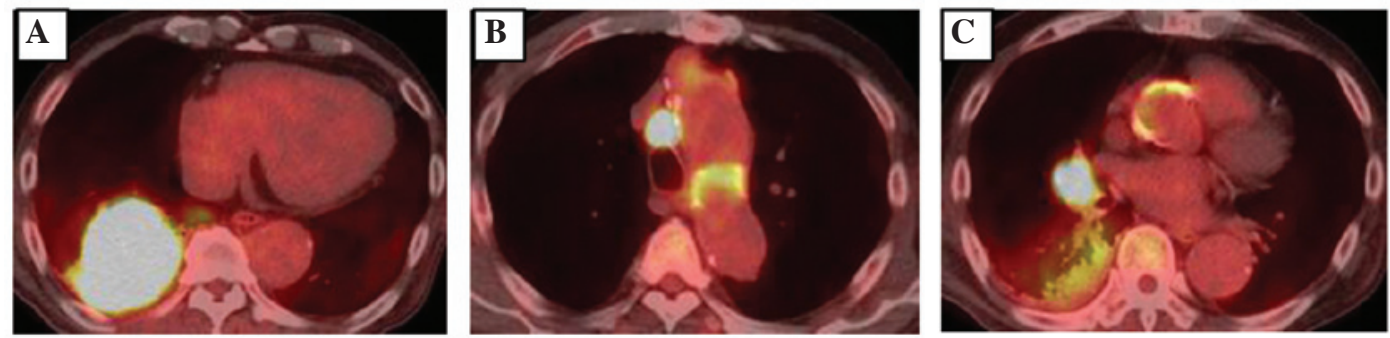

Figure 2. Positron emission tomography scan performed at the time of the referral, revealing a high uptake of fluorodeoxyglucose in the mass in (A) the right lower lobe and (B) the mediastinal lymph nodes.(C) The uptake in the right-sided consolidation adjacent to the pleura was low.

Center General Hospital as a novel mass in the right lower lobe had appeared and rapidly increased in size, which was accompanied by elevation of soluble interleukin- 2 receptor (sIL2R; Fig. 1D and E).

Physical examination revealed that respiratory sounds were decreased in the right lower lung. A chest radiograph revealed a mass and consolidation in the right lower lung. A computed tomography scan revealed an expanding mass and consolidation in a region of emphysema adjacent to the pleura in the right lower lobe (Fig. 1E). A positron emission tomography scan revealed high uptake of fluorodeoxyglucose in the mass in the right side of the lung [maximum standardized uptake value $\left.\left(\mathrm{SUV}_{\max }\right), 24.3\right]$ and mediastinal lymph nodes $\left(\mathrm{SUV}_{\max }, 18.2\right)$ but the uptake was low in the consolidation region $\left(\mathrm{SUV}_{\max }, 3.5\right)$ (Fig. 2). Laboratory examinations revealed a white blood cell count of $12400 \mathrm{cells} / \mathrm{mm}^{3}$, comprising $82 \%$ neutrophils, a C-reactive protein level of $6.4 \mathrm{mg} / \mathrm{dl}$, a neuron-specific enolase level of $19.2 \mathrm{ng} / \mathrm{ml}$, a progastrin-releasing peptide level of $33.8 \mathrm{pg} / \mathrm{ml}$, a carcinoembryonic antigen level of $5.4 \mathrm{ng} / \mathrm{ml}$, a cytokeratin 19 fragment 

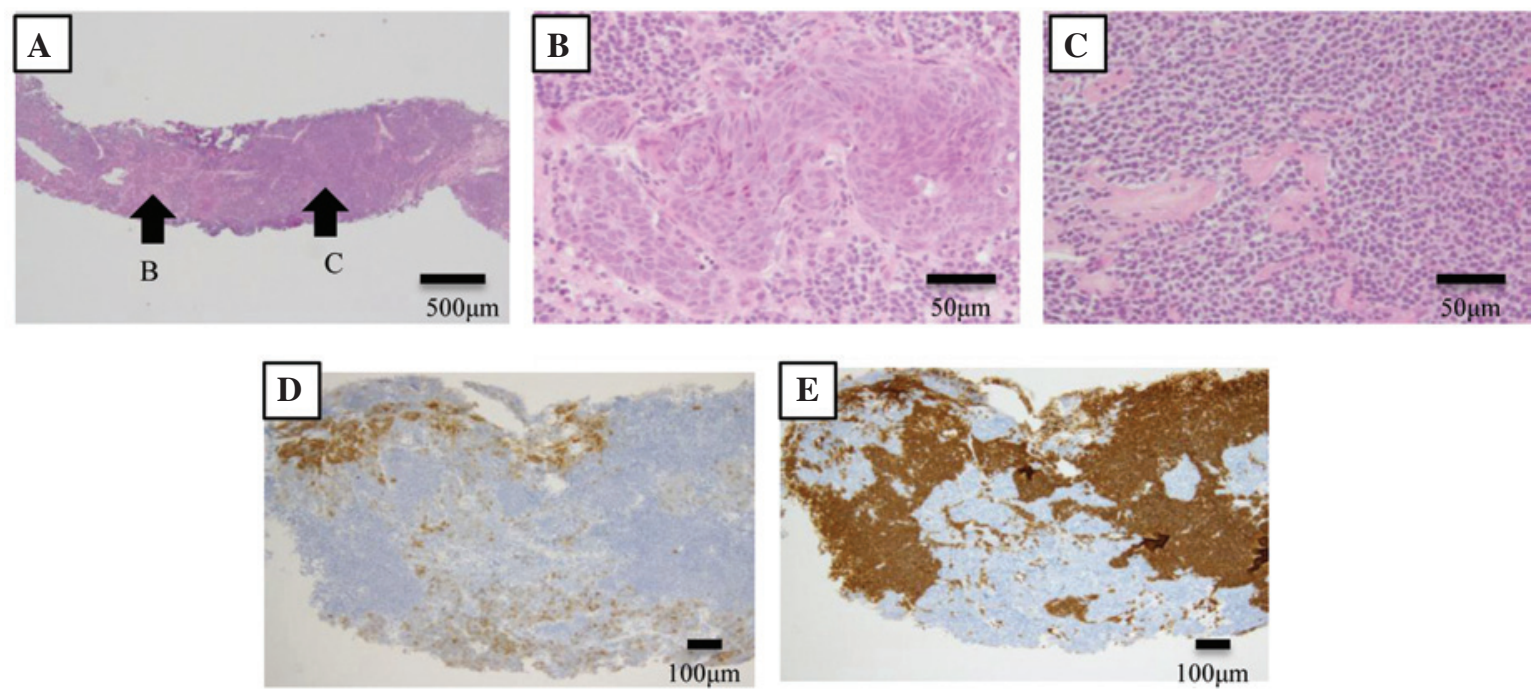

Figure 3. (A) Histological examination of the biopsy of the lung mass and consolidation subsequent to hematoxylin and eosin staining revealed two types of cellular abnormalities. (B) One was carcinoma with a rosette pattern, and (C) the other was an aggregation of small lymphocytes. (D) Certain areas of the tumor were posistive for cluster of differentiation (CD) 56 staining, and (E) staining for CD20 was positive in other areas.

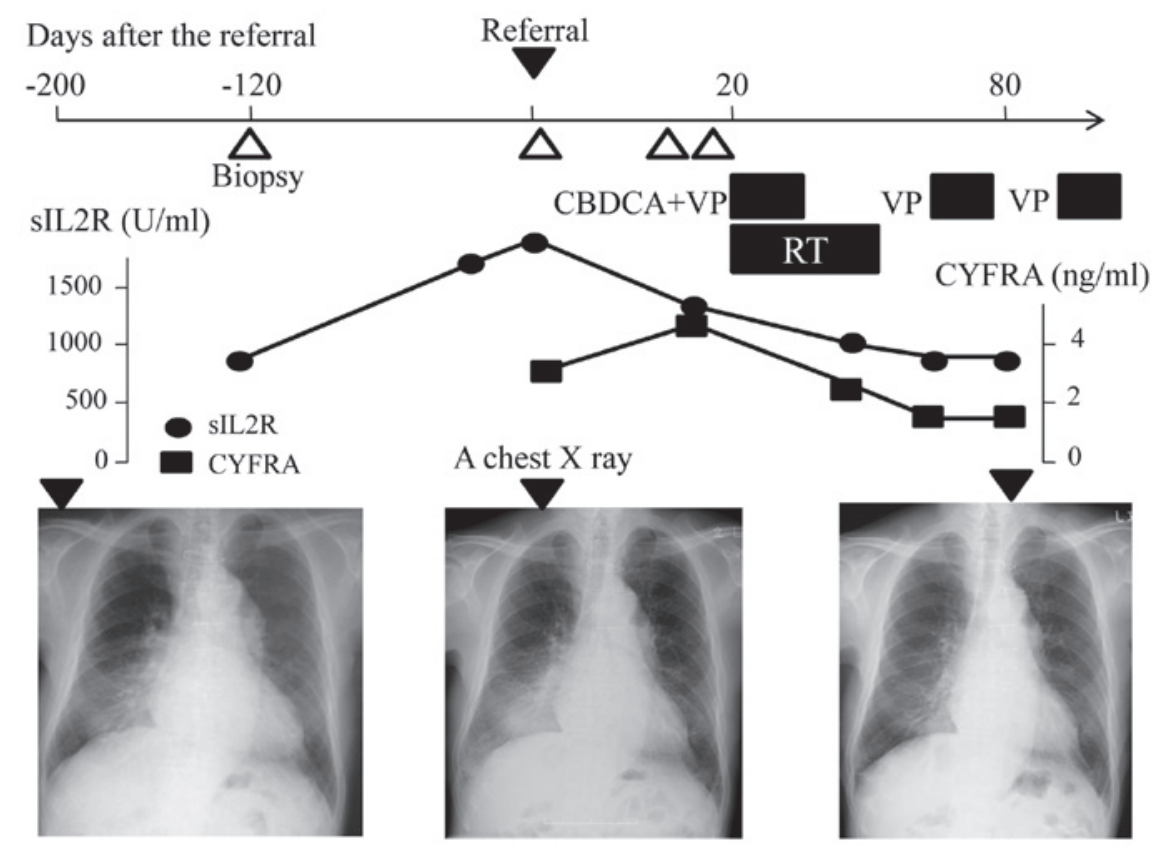

Figure 4. Clinical course in the treatment of the present patient. CBDCA, carboplatin; VP, etoposide; sIL2R, soluble interleukin-2 receptor; CYFRA, cytokeratin 19 fragment; RT, radiation therapy.

level of $3.4 \mathrm{ng} / \mathrm{ml}$, a squamous cell carcinoma-related antigen level of $2.1 \mathrm{ng} / \mathrm{ml}$, and a sIL2R level of 1756 units $/ \mathrm{ml}$. Histological examination of repeat bronchoscopic and echo-guided biopsies of the right mass and consolidation revealed two types of abnormal cells (Fig. 3A). One cell type was carcinoma exhibiting a rosette pattern (Fig. 3B), which was positive for cytokeratin and cluster of differentiation (CD)56 expression, while the other cell type was aggregated small lymphocytes (Fig. 3C) that were CD20-positive but CD3-negative. Certain regions were positive for CD56 expression, while other areas in the tumor were positive for CD20 expression (Fig. 3D and E), which was considered a mix of neuroendocrine carcinoma, either LCNEC or small cell carcinoma, and MALT lymphoma. Endobronchial ultrasound-guided transbronchial needle aspiration was performed on the swollen $4 \mathrm{R}$ lymph node to determine the stage of the lesion. The aspiration revealed a medium to large-sized endocrine carcinoma, with a rosette pattern that was diffusely cytokeratin-positive and did not express CD20 and CD56. Therefore, it was hypothesized that the lesion resulted from the metastasis of LCNEC. The patient was diagnosed with LCNEC at a clinical tumor-node-metastasis stage (cTNM) of cT2bN2M0 (stage 3A) and MALT lymphoma at Ann Arbor stage 1E.

Chemotherapy, consisting of carboplatin (area under the curve, 4 ; day 1) and etoposide $\left(80 \mathrm{mg} / \mathrm{m}^{2}\right.$; days $\left.1-3\right)$, 

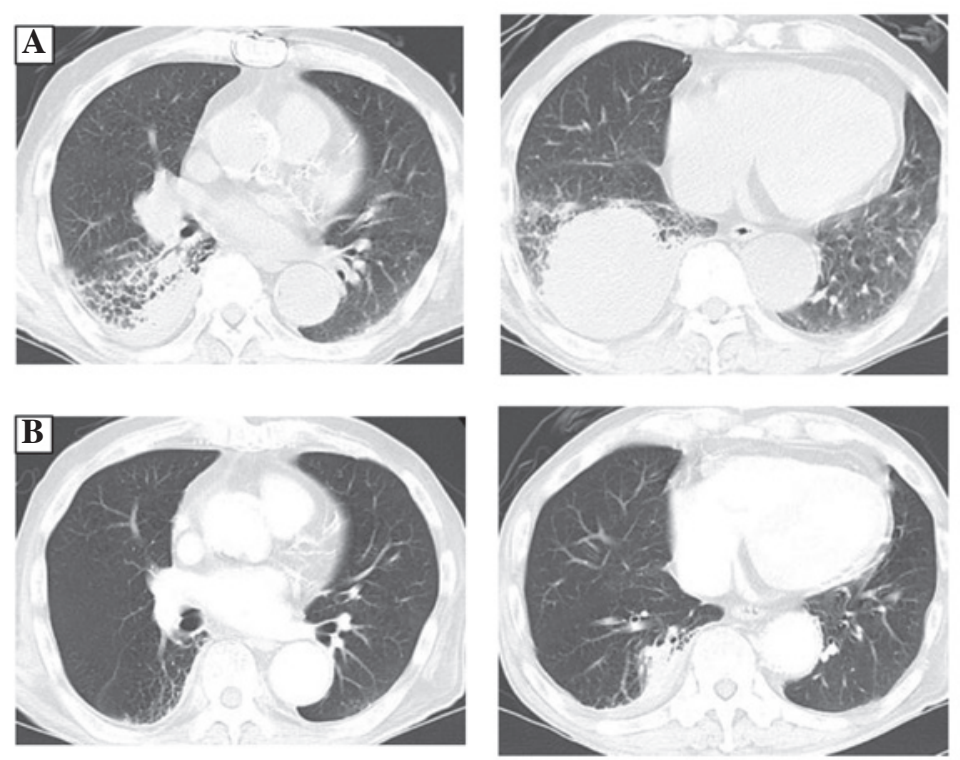

Figure 5. Computed tomography scan of the chest revealing a significant reduction in the size of the mass and consolidation adjacent to the pleura in the right lower lobe (A) prior to treatment and (B) three months after the initiation of chemoradiotherapy.

combined with radiation therapy (45 Gy; 30 fractions) were commenced, which markedly affected the mass and consolidation (Fig. 4). Subsequent to four cycles of chemotherapy, the mass was significantly reduced in size and the consolidation was also reduced (Fig. 5). The effect was considered to be a near-complete response, which continued for 144 days. Subsequently, liver and bone metastases appeared and the patient declined further chemotherapy. Although the lung tumor and consolidation did not enlarge, the metastatic lesions increased in size. Eight months subsequent to referral, the patient succumbed to hepatic failure caused by liver metastasis.

\section{Discussion}

The present study, to the best of our knowledge, reports the first case of a patient with combined LCNEC and MALT lymphoma of the lung that responded well to chemoradiotherapy and was followed over six years.

MALT lymphoma is a low grade B cell lymphoma that mostly occurs in the gastrointestinal tract, accounting for only $0.5 \%$ of lung tumors. Therefore, pulmonary MALT lymphoma is extremely rare $(2,3)$. LCNEC is a tumor that is regarded as a variant of large cell carcinoma by the World Health Organization. LCNEC is also rare, accounting for $2.4 \%$ of lung cancers. The biological features of LCNEC are similar to those of small cell carcinoma and the prognosis is extremely poor $(8,9)$. Surgery is performed to treat LCNEC, if possible, but the current results are not satisfactory. Although a standard therapy for LCNEC has not been established, chemoradiotherapy similar to that administered for the treatment of small cell carcinoma is administered to patients with inoperable LCNEC (11).

It has been speculated that MALT lymphoma of the lung is associated with chronic respiratory infection, chronic inflammatory diseases, autoimmune diseases or antigen exposure, such as exposure to tobacco $(12,13)$. The median time to progression for pulmonary MALT lymphoma has been reported to be 5.6 years, and the prognosis was the same regardless of whether treatment consisted of chemotherapy or surgery $(14,15)$. In addition, Troch et al (6) reported that pulmonary MALT lymphoma may not require immediate treatment in asymptomatic patients and thus, a watch-and-wait strategy may be adopted.

Cases of combined MALT lymphoma and gastric cancer have been reported frequently. However, the combination of lung cancer with MALT lymphoma is rare and few cases have been reported at present (16-21). To the best of our knowledge, the combination of LCNEC and MALT lymphoma has been reported in only one other case (18), in which the patient suffered from sarcoidosis. Surgery was performed for the treatment of a mass shadow in the left lower lobe of the lung, and the concurrent occurrence of LCNEC and MALT lymphoma was an incidental finding. In the present case, the consolidation adjacent to the pleura was thought to be MALT lymphoma and the novel mass was LCNEC, since the consolidation appeared initially and increased in size extremely slowly, whereas the mass appeared later and rapidly became enlarged.

As the combination of lung cancer with MALT lymphoma is rare, the mechanism is not well-elucidated. However, possible causes of the formation of MALT lymphoma and lung cancer were reported to be either associated with the API2-MALT1 fusion gene or smoking $(16,17)$. By contrast, when the rate of combination of malignant lymphoma with other types of cancer was statistically analyzed, the combination with lung cancer was reported to be a coincidental occurrence (22). The present patient was followed for six years, and this observation may indicate that stimulation from the MALT lymphoma contributed to the formation of lung cancer. However, there is insufficient data on the combination of lung cancer with MALT lymphoma. This concept requires further elucidation through the examination of additional cases.

In conclusion, the present study reported the case of a patient with combined LCNEC and MALT lymphoma of the lung that was followed over six years, including the onset of the disease. 


\section{References}

1. Isaacson P and Wright DH: Malignant lymphoma of mucosa-associated lymphoid tissue. A distinctive type of B-cell lymphoma. Cancer 52: 1410-1416, 1983

2. Du MQ: MALT lymphoma: recent advances in aetiology and molecular genetics. J Clin Exp Hematop 47: 31-42, 2007.

3. Chilosi M, Zinzani PL and Poletti V: Lymphoproliferative lung disorders. Semin Respir Crit Care Med 26: 490-501, 2005.

4. Cadranel J, Wislez M and Antoine M: Primary pulmonary lymphoma. Eur Respir J 20: 750-762, 2002.

5. Addis BJ, Hyjek E and Isaacson PG: Primary pulmonary lymphoma: a re-appraisal of its histogenesis and its relationship to pseudolymphoma and lymphoid interstitial pneumonia. Histopathology 13: 1-17, 1988.

6. Troch M, Streubel B, Petkov V, Turetschek K, Chott A and Raderer M: Does MALT lymphoma of the lung require immediate treatment? An analysis of 11 untreated cases with long-term follow-up. Anticancer Res 27: 3633-3637, 2007.

7. Travis WD, Linnoila RI, Tsokos MG, et al: Neuroendocrine tumors of the lung with proposed criteria for large-cell neuroendocrine carcinoma. An ultrastructural, immunohistochemical and flow cytometric study of 35 cases. Am J Surg Pathol 15: 529-553, 1991.

8. Iyoda A, Hiroshima K, Toyozaki T, Haga Y, Fujisawa T and Ohwada H: Clinical characterization of pulmonary large cell neuroendocrine carcinoma and large cell carcinoma with neuroendocrine morphology. Cancer 91: 1992-2000, 2001.

9. Iyoda A, Hiroshima K, Nakatani Y and Fujisawa T: Pulmonary large cell neuroendocrine carcinoma: its place in the spectrum of pulmonary carcinoma. Ann Thorac Surg 84: 702-707, 2007.

10. Iyoda A, Hiroshima K, Moriya Y, Sekine Y, Shibuya K, Iizasa T, Nakatani Y and Fujisawa T: Prognostic impact of large cell neuroendocrine hisotology in patients with pathologic stage Ia pulmonary non-small cell carcinoma. J Thorac Cardiovasc Surg 132: 312-315, 2006.

11. Fujiwara Y, Sekine I, Tsuta K, et al: Effect of platinum combined with irinotecan or paclitaxel against large cell neuroendocrine carcinoma of the lung. Jpn J Clin Oncol 37: 482-486, 2007.

12. Thieblemont C, Bastion Y, Berger F, et al: Mucosa-associated lymphoid tissue gastrointestinal and nongastrointestinal lymphoma behavior: analysis of 108 patients. J Clin Oncol 15: 1624-1630, 1997.
13. Stagnaro E, Tumino R, Parodi S, et al: Non-Hodgkin's lymphoma and type of tobacco smoke. Cancer Epidemiol Biomarkers Prev 13: 431-437, 2004.

14. Oh SY, Kim WS, Kim JS, et al: Pulmonary marginal zone B-cell lymphoma of MALT type - what is a prognostic factor and which is the optimal treatment, operation, or chemotherapy?: Consortium for Improving Survival of Lymphoma (CISL) study. Ann Hematol 89: 563-568, 2010.

15. Thieblemont C, Berger F, Dumontet C, et al: Mucosa-associated lymphoid tissue lymphoma is a disseminated disease in one third of 158 patients analyzed. Blood 95: 802-806, 2000.

16. Ichihara E, Tabata M, Takigawa N, et al: Synchronous pulmonary MALT lymphoma and pulmonary adenocarcinoma after metachronous gastric MALT lymphoma and gastric adenocarcinoma. J Thorac Oncol 3: 1362-1363, 2008.

17. Suzuki T, Akizawa T, Suzuki H, Kitazume K, Omine M and Mitsuya T: Primary tracheal mucosa-associated lymphoid tissue lymphoma accompanying lung cancer. Common tumorigenesis or coincidental coexistence? Jpn J Thorac Cardiovasc Surg 48: $817-819,2000$

18. Itoh T, Kobayashi D, Shiratuchi N, Rensha K and Minami K: Case of overlapping cancers complicated with sarcoidosis. Nihon Kokyuki Gakkai Zasshi 47: 410-414, 2009 (In Japanese).

19. Chanel S, Burke L, Fiche M, et al: Synchronous pulmonary adenocarcinoma and extranodal marginal zone/low-grade B-cell lymphoma of MALT type. Hum Pathol 32: 129-132, 2001.

20. Jung CY and Kwon KY: A case of synchronous lung adenocarcinoma and extranodal marginal zone B-cell lymphoma of mucosa-associated lymphoid tissue (MALT) type. Tuberc Respir Dis (Seoul) 73: 61-66, 2012.

21. Adrish M, Venkatram S,Niazi M and Diaz-Fuentes G: Concurrent lung squamous cell carcinoma and extranodal marginal zone B-cell lymphoma of mucosa-associated lymphoid tissue type. J Bronchology Interv Pulmonol 21: 96-99, 2014.

22. Tihan T and Filippa DA: Coexistence of renal cell carcinoma and malignant lymphoma. A causal relationship or coincidental occurrence? Cancer 77: 2325-2331, 1996. 